\title{
OCORRÊNCIA DE DIABETES MELLITUS GESTACIONAL EM UMA UNIDADE BÁSICA DE SAÚDE DE CAJAZEIRAS-PB NO ANO DE 2015
}

\author{
OCCURRENCE OF DIABETES MELLITUS GESTATIONAL IN A BASIC \\ UNIT OF HEALTH OF CAJAZEIRAS-PB IN THE YEAR 2015.
}

\author{
Bruna Vieira Lins Campos ${ }^{1}$ \\ Wigna Luana de Figueirêdo Pimenta ${ }^{2}$ \\ Valdez Vieira da Silva ${ }^{3}$ \\ Vládia Maria de Souza Albuquerque ${ }^{4}$ \\ Murilo Rodrigues Pereira da Silva ${ }^{5}$ \\ Maria Algeni Tavares Landim ${ }^{6}$
}

RESUMO: Objetivo: Verificar a ocorrência de Diabetes Mellitus Gestacional em uma unidade básica de saúde de Cajazeiras-PB. Metodologia: Trata-se de uma pesquisa de campo, tipo descritiva, com uma abordagem quali-quantitativa. A população estudada foi composta por gestantes que fizeram o pré-natal em uma unidade básica de saúde de Cajazeiras-PB, no ano de 2015. Foram avaliados prontuários de 100 gestantes que realizaram o pré-natal nesta unidade. Resultados: Dos prontuários avaliados, apenas 2 mostraram diagnóstico de Diabetes Mllitus Gestacional (DMG) com valores superiores à $126 \mathrm{mg} / \mathrm{dL}$ de Glicemia de Jejum, e os 98 restantes apresentaram valores inferiores à $92 \mathrm{mg} / \mathrm{dL}$. De acordo com os dados coletados, observou-se que a prevalência do DMG na Unidades Básica de Saúde de cajazeiras-PB apresentou uma frequência de 2 casos apenas e em nenhum momento as gestantes tiveram acompanhamento nutricional. O nutricionista tem papel fundamental na prevenção e tratamento do DMG, juntamente com outros profissionais da área da saúde, com o objetivo de controlar a glicemia e evitar problemas futuros tanto para a mãe como para o bebê. Conclusão: Os resultados encontrados apontam que os perfis glicêmicos da maioria das gestantes das unidades básicas de saúde Cajazeiras-PB, encontram-se dentro dos valores de normalidade. Esse resultado mostra que as gestantes estão fazendo a prevenção, se alimentando da forma adequada. A presente pesquisa é relevante, pois se trata de uma patologia que coloca em risco a saúde da gestante e do feto. Determinar a

\footnotetext{
${ }^{1}$ Biomédica formada na Faculdade Santa Maria - FSM, Cajazeiras - PB.

2 Biomédica formada na Faculdade Santa Maria - FSM, Cajazeiras - PB.

${ }^{3}$ Biomédica formada na Faculdade Santa Maria - FSM, Cajazeiras - PB.

${ }^{4}$ Biomédica formada na Faculdade Santa Maria - FSM, Cajazeiras - PB.

${ }^{5}$ Biomédica formada na Faculdade Santa Maria - FSM, Cajazeiras - PB.

${ }^{6}$ Biomedicina Especialista - Faculdade Leão Sampaio -Unileão - Juazeiro do Norte - Ce.
} 
ocorrência de DMG irá evidenciar a necessidade do controle e da prevenção à população sobre essa doença, buscando assim uma melhor qualidade de vida para as futuras mães e seus filhos.

Palavras chave: Diabetes mellitus gestacional. Prevalência. Diagnóstico.

ABSTRACT: Objective: To verify the occurrence of Gestational Diabetes Mellitus in a basic health unit of Cajazeiras-PB. Methodology: This is a field research, descriptive type. This research has a qualitative-quantitative approach. The study population consisted of pregnant women who underwent prenatal care at a basic health unit in Cajazeiras-PB, in the year 2015. The medical records of 100 pregnant women who underwent prenatal care at this unit were evaluated. Results: Only two showed a diagnosis of Gestational Diabetes with values greater than $126 \mathrm{mg} / \mathrm{dl}$ of Fasting Glycemia, and the remaining 98 presented values below $92 \mathrm{mg} / \mathrm{dl}$. According to the data collected, it was observed that the prevalence of DMG in the Basic Health Units of PB-box showed a frequency of 2 cases only and at no time did the pregnant women receive nutritional monitoring. The nutritionist plays a key role in the prevention and treatment of GDM, along with other health professionals, in order to control glycemia and prevent future problems for both mother and baby. Conclusion: The results found that the glycemic profiles of the majority of the pregnant women of the basic health units Cajazeiras- $P B$, are within the values of normality. This result shows that the pregnant women are doing the prevention, feeding themselves properly. The present research is relevant because it is a pathology that puts at risk the health of the pregnant woman and the fetus. Determining the occurrence of DMG will highlight the need for population control and prevention of this disease, thus seeking a better quality of life for future mothers and their children.

Keywords: Gestational diabetes mellitus. Prevalence. Diagnosis. 


\section{INTRODUÇÃO}

O Diabetes Gestacional se constitui na elevação da glicemia detectada na gestação, geralmente essa condição é diagnosticada com exames provocativos que empregam sobrecarga de glicose. Atualmente os profissionais da saúde sugerem a detecção precoce de gestantes com elevado risco no primeiro atendimento do prénatal, isso faz com que sejam encontrados casos de diabetes existentes ou prestes a serem desenvolvidos (WEINERT et al., 2011).

Durante a gestação acontecem diversas mudanças no organismo e uma delas é a modificação do comando da glicose e utilidade da insulina, essas alterações ocorrem devido o aumento dos hormônios gerados pela placenta, que são: progesterona, estrogênio e gonadotrofinas, sendo esta última, responsável por impedir o desempenho da insulina, aumentando assim a possibilidade da gestante desenvolver o DMG, esse processo normalmente se estabelece entre a vigésima e vigésima quarta semana de gestação (LIMA; BRASILEIRO; ROSA, 2012).

Existem alguns elementos de risco para o surgimento de DMG, são eles: $A$ gestante ter idade igual ou superior a 25 anos, histórico familiar de diabetes em parentes do $1^{\circ}$ grau, antecedentes obstétricos (macrossomia, morte fetal ou neonatal de causa desconhecida, perdas gestacionais de repetição, diabetes gestacional), sobrepeso, obesidade, crescimento fetal excessivo, hipertensão ou pré-eclâmpsia, deposição central excessiva de gordura corporal, síndrome de ovários policísticos (SOUSA et al., 2014).

Reconhecer o DMG é de extrema importância e deve acontecer o mais precoce possível, pois seu diagnóstico propõe diminuir os efeitos adversos dessa desordem metabólica com relação mãe-filho, como também diferenciar as mulheres com risco elevado de desenvolver diabetes no futuro (NOGUEIRA et al., 2011).

$\mathrm{O}$ auxílio pré-natal deve dar prioridade a precauções relevantes, tais como: auxiliar na dieta ideal para a gestante, mostrar a importância da atividade física, avaliar periodicamente a glicemia da gestante e no caso de detecção do DMG 
instruir sobre o tratamento de forma a evitar resultados prejudiciais para a gravida e o recém-nascido, tudo isso para que o quadro não evolua para uma desordem metabólica e possível cetoacidose, infecção urinária de recorrências com risco de infecção renal aguda e surgimento simultâneo da hipertensão que pode causar aborto, como também nascimento do bebê antes das 37 semanas de gestação e perturbações no desenvolvimento e no nascimento (NETA et al., 2014).

Este estudo tem como objetivo avaliar o perfil glicêmico das gestantes, atendidas em uma unidade básica de saúde de Cajazeiras-PB.

\section{METODOLOGIA}

Trata-se de uma pesquisa de campo, tipo descritiva, com uma abordagem quali-quantitativa.

A população estudada foi composta por 100 gestantes, que fizeram o prénatal em uma unidade básica de saúde de Cajazeiras-PB, no ano de 2015, os dados foram coletados no mês de outubro de 2016, o material utilizado para a coleta de dados dessa pesquisa foram os prontuários, que continham os resultados dos exames incluindo o de glicemia, idade e peso das gestantes participantes da UBS da cidade de Cajazeiras - PB.

Os dados obtidos foram organizados em gráficos do Software excel versão 2010, usando estatística descritiva, onde os resultados foram mostrados afim da compreensão do leitor.

O projeto de pesquisa, foi submetido e aprovado pelo Comitê de Ética e Pesquisa da Faculdade Santa Maria na Cidade de Cajazeiras-PB, conforme o parecer, tendo o número 1.763.229. A pesquisa foi norteada a partir de normas e diretrizes que obedecem à resolução 466/12. 


\section{RESULTADOS E DISCUSSÃO}

Foram avaliados prontuários de 100 gestantes, que realizaram o pré-natal em uma Unidades Básica de Saúde de cajazeiras-PB, destes, apenas 2 casos mostraram diagnóstico de Diabetes Gestacional, com valores superiores a $126 \mathrm{mg} / \mathrm{dl}$ de Glicemia de Jejum, o valor glicêmico encontrado no prontuário dessas gestantes foram, $145 \mathrm{mg} / \mathrm{dL}$ e $162 \mathrm{mg} / \mathrm{dL}$, e os 98 prontuários restante apresentaram valores inferiores à $92 \mathrm{mg} / \mathrm{dL}$.

Dentre as pacientes diabéticas, foi avaliado se o fator idade poderia ser relevante na prevalência do DMG, observando-se que nenhuma gestante com o diagnóstico de diabetes apresentou idade de 15 a 20 anos. Os casos ocorreram em gestantes com 27 e 31 anos, demonstrando que o fator idade pode ser considerado fator de risco para a patologia.

Gráfico 1: Idade das gestantes de uma unidade básica de saúde de Cajazeiras, PB, no ano de 2015.

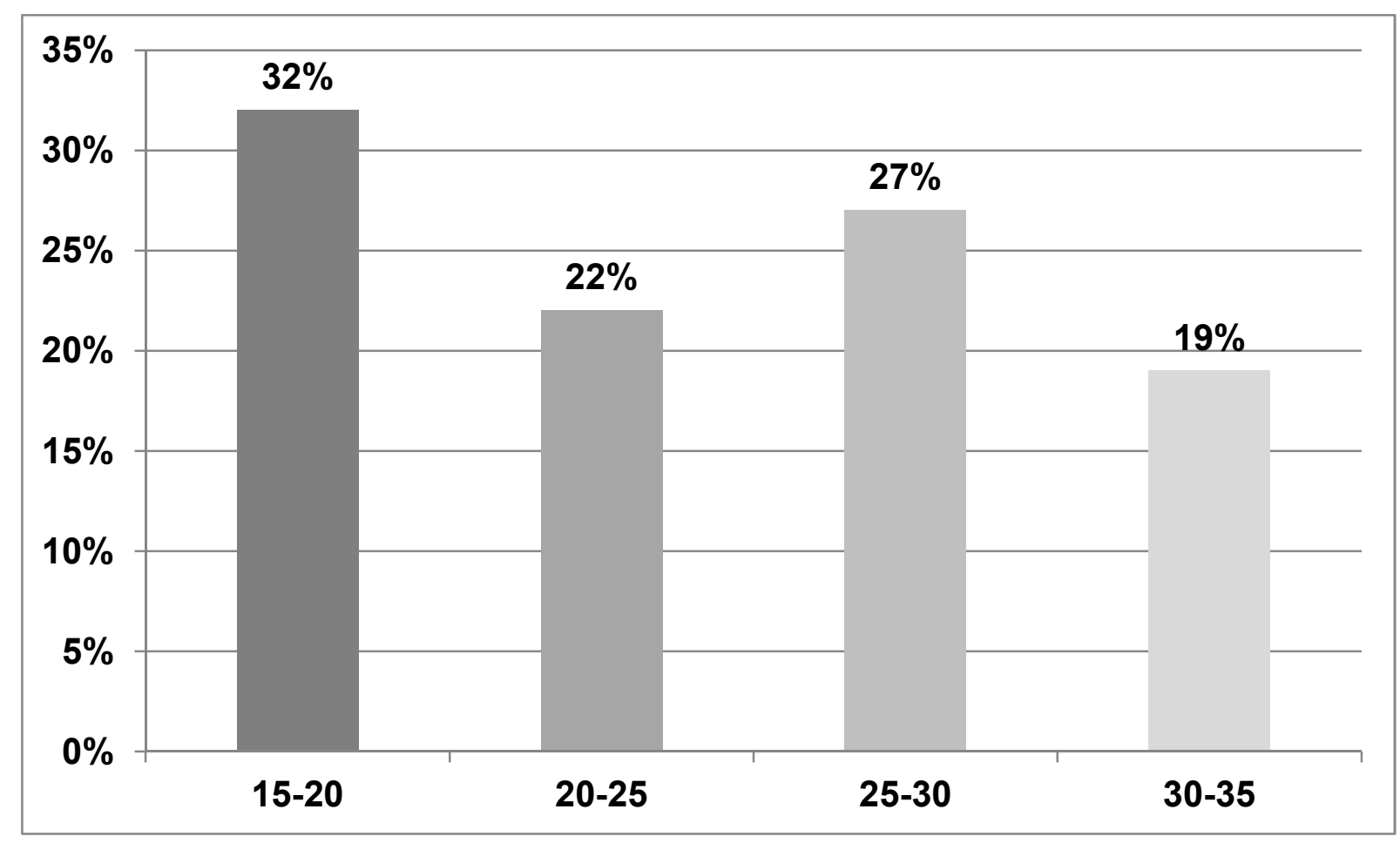


Esses dados comprovam que o DMG ocorre mais em mulheres com idade acima de 20 anos. Corroborando com a análise feita por Padilha e colaboradores (2010) e Neta (2014), onde afirma que o Diabetes Mellitus Gestacional predomina em mulheres com idade maior que 20 anos, atendidas em serviços de pré-natal do (SUS), ocorrendo então em 7,6\% delas, sendo que $94,0 \%$ dos eventos mostraram somente tolerância diminuída à glicose e $6,0 \%$ apresentaram o aumento da glicemia semelhante ao grau de diabetes fora período da gravidez. No trabalho de Neta (2014), foi diagnosticado o DMG em $60,9 \%$ de mulheres com mais de 30 anos assim revelando que o fator idade pode ser considerado um fator de risco.

No trabalho de Schmitt (2009), constatou que o fator idade, isoladamente, não pôde ser considerado como risco para o desenvolvimento do diabetes gestacional.

Em relação ao peso, de acordo com os dados dos prontuários, as 2 gestantes apresentaram peso maior que $75 \mathrm{Kg}$, onde uma pesava 78 e a outra $85 \mathrm{~kg}$. Tendo em vista que o peso elevado também é um fator de risco para o DMG.

O peso descrito também foi um dado coletado em prontuários, porém, em nenhum dos casos analisados havia a informação da altura da gestante, o que dificultou a interpretação do peso e do ganho de peso gestacional. No entanto, foi observado que houve maior frequência de diabetes gestacional naquelas mulheres que tiveram o peso superior a $75 \mathrm{~kg}$ no momento da consulta, com isso podemos observar que, quanto maior o ganho de peso gestacional, maior a incidência do diabetes gestacional. 
Gráfico 2: Peso das gestantes de uma unidade básica de saúde de Cajazeiras, $\mathrm{Pb}$, no ano de 2015.

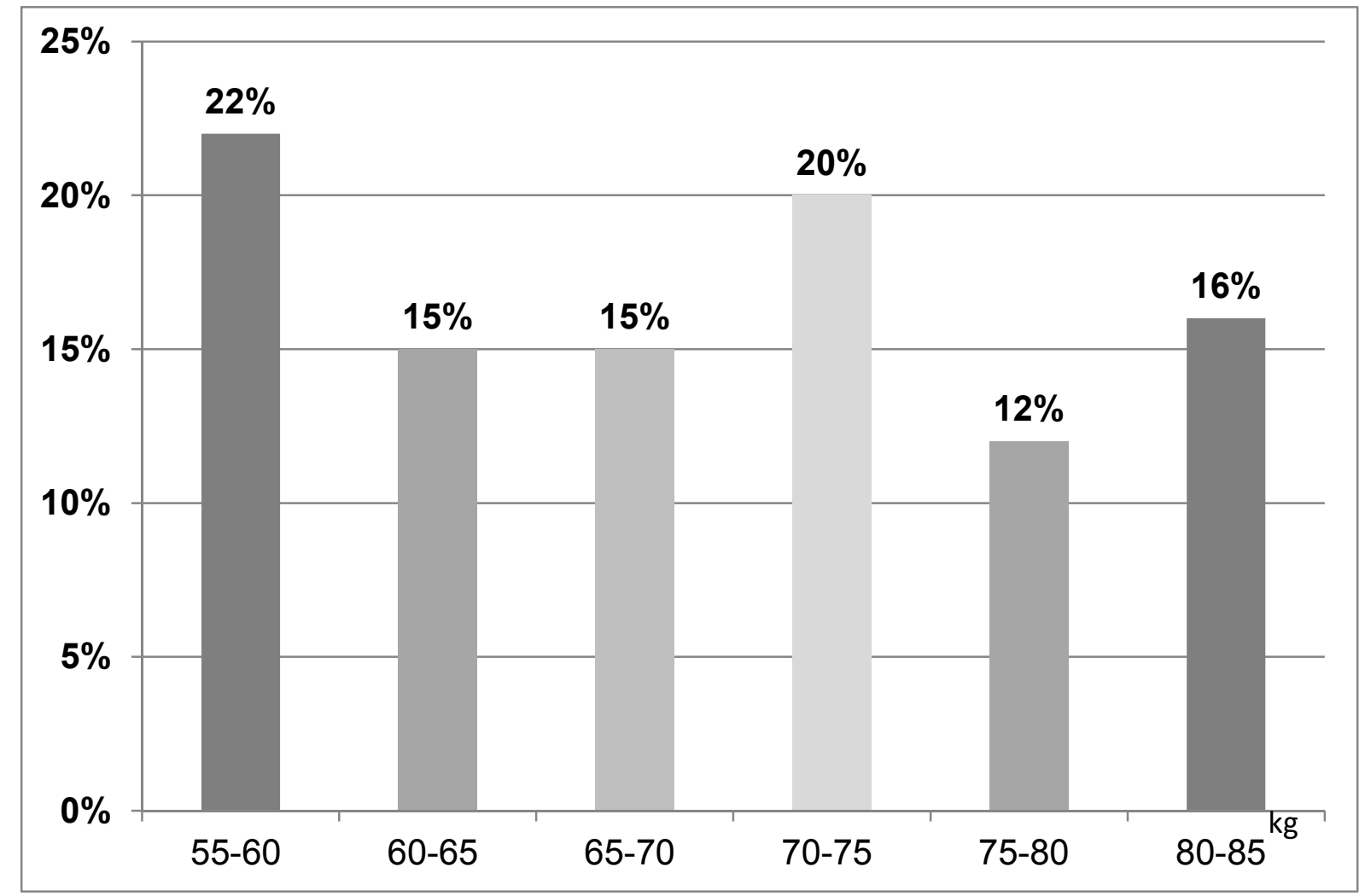

Os achados neste trabalho correlacionam com o trabalho de Oliveira (2014) e Hillier (2008), que demonstraram forte associação entre obesidade e diabetes gestacional. Apesar de não ser possível a classificação de obesidade nas gestantes, pode-se inferir que o excesso de ganho de peso correlaciona com o DMG. 0 excesso de peso associa-se fortemente à condições clínicas, como o DMG, confirmando a literatura que relaciona o aumento da incidência de DMG em mulheres obesas.

Ainda há controvérsias quanto ao valor ideal da glicemia de jejum no rastreamento do DMG. Esse dado varia de acordo com o valor empregado e com a população estudada. Foi utilizado para a busca da patologia em questão, o valor abaixo de $92 \mathrm{mgldL}$, onde é considerado valor normal e acima de $126 \mathrm{mgldL}$ para glicemia alterada. 
Sabemos que o exame de glicemia de jejum é o mais utilizado para detecção do DMG, principalmente nas unidades básicas de saúde, por ser um exame mais acessível em relação ao preço e de fácil execução.

Gráfico 3: Valor glicêmico das gestantes de uma unidade básica de saúde de Cajazeiras, PB, no ano de 2015.

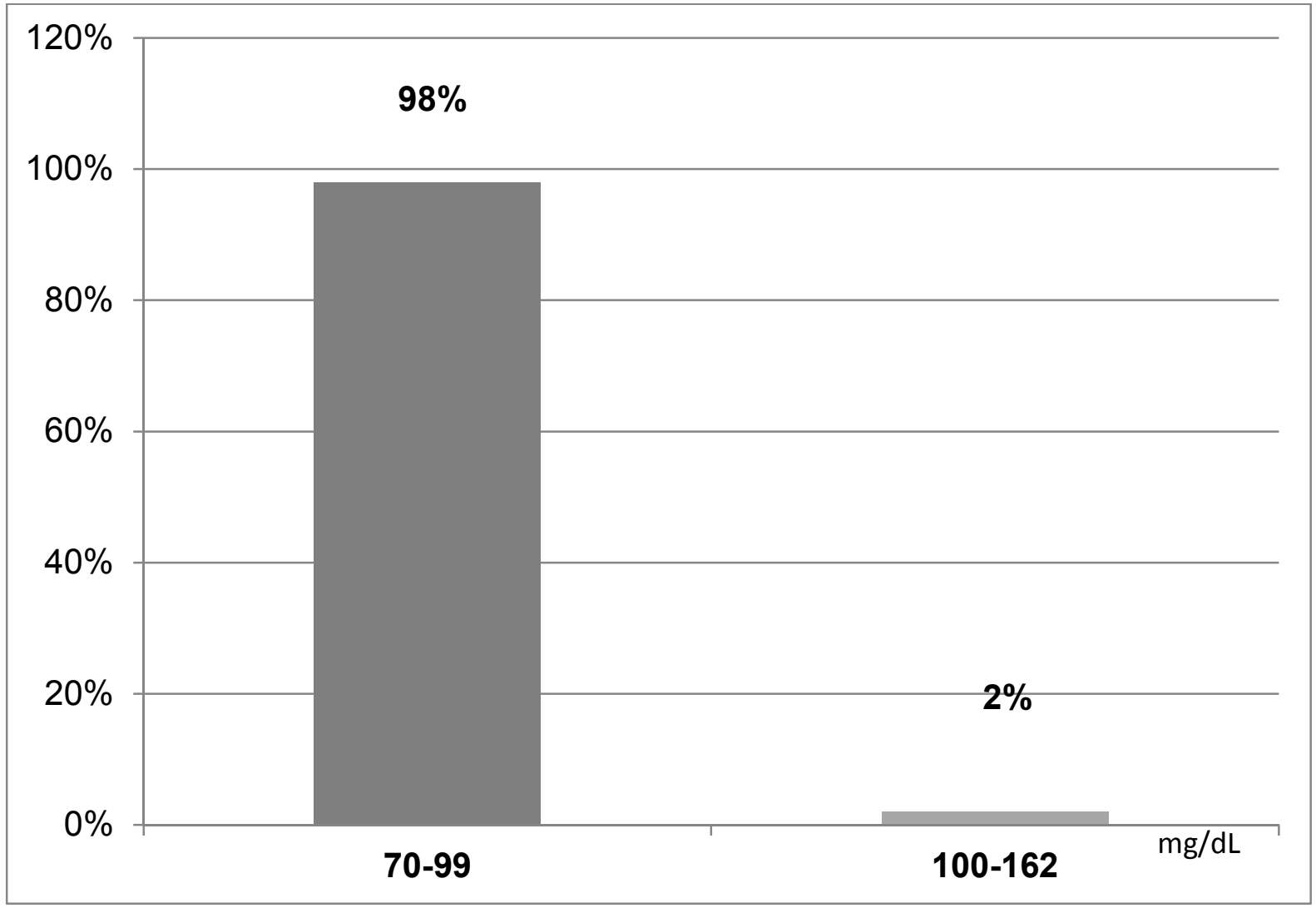

Nesse estudo foi considerado DMG se a glicemia da gestante tivesse $\geq 126$ $\mathrm{mg} / \mathrm{dL}$ e normal $92 \mathrm{mg} / \mathrm{dL}$ assim corroborando com a análise de Oliveira e colaboradores (2014) ao realizar o exame de glicemia de jejum na gestante, se o valor achado for $\geq 126 \mathrm{mg} / \mathrm{dl}$ é reconhecido o DMG pré-gestacional, mas sempre deve ser feita novamente a glicemia de jejum para confirmatório dessa condição, já que sabemos que existem alguns interferentes, como se a gestante não tiver feito o jejum de 12 horas, assim como erros laboratoriais. Se o exame mostrar valor < 92 $\mathrm{mg} / \mathrm{dL}$, a gestante deve ser avaliada novamente no segundo trimestre da gestação.

Em relação ao período de gestação, as duas gestantes tiveram a patologia diagnosticada no $3^{\circ}$ trimestre, concordando com Sousa (2014), Queiroz (2016) e 
Bolognani (2011), onde a maioria das mulheres apresentou a DMG entre 24 e 27 semanas de gestação, é a partir dai que ela se manifesta, havendo tempo bastante para a conduta terapêutica exercer seus efeitos.

\section{CONCLUSÃO}

Com base neste estudo podemos concluir, que a prevalência do DMG, em relação às gestantes, atendidas em uma Unidades Básica de Saúde de CajazeirasPB, foi de 2 casos em 100 gestantes contidas nos prontuários a partir das coletas de dados, os fatores de risco que parecem estar associados à ocorrência do DMG, são idade da gestante e peso, conforme o período de diagnóstico, as gestantes tiveram a patologia diagnosticada no terceiro trimestre gestacional.

Os resultados encontrados apontam que os perfis glicêmicos da maioria das gestantes das unidades básicas de saúde Cajazeiras-PB, encontram-se normais, mostrando que as gestantes estão fazendo a prevenção e se alimentando da forma adequada.

\section{REFERÊNCIAS BIBLIOGRÁFICAS}

BOLOGNANI, Cláudia Vicari; SOUZA, Sulani Silva de; CALDERON, Iracema de Mattos Paranhos. Diabetes mellitus gestacional. Enfoque nos novos critérios diagnósticos: Gestational diabetes mellitus - focus on new diagnostic criteria. Com. Ciências Saúde, Brasília-brasil, v. 22, n. 1, p.531-542, 2011.

HILLIER ta, PEDULA kl, VESCO kk, SCHMIDT mm, MULLEN ja, LEBLANC es, et al. Excess gestational weightgain: modifying fetal macro-somia risk associated with maternal glucose. Obstetrics \&Gynecology. 2008;11(5):1007-14.11.

LIMA, Daliane Angelica; BRASILEIRO, Aline Alves; ROSA, Lorena Pereira de Souza. Riscos e consequências das diabetes gestacional: uma revisão bibliográfica. Estudos, Goiânia, v.39. n. 4. p.561-567, out. 2012.

NETA, Francisca Adriele Vieira et al. Avaliação do perfil e dos cuidados no pré-natal de mulheres com diabetes mellitus gestacional. Revista Rene, Fortaleza, Ce, Brasil, v.15, n. 5, p.823-831, 28 out. 2014.

NOGUEIRA, Anelise Impeliziere et al. Diabetes Gestacional: perfil e evolução de um grupo de pacientes do Hospital das Clínicas da UFMG. Revista de Medicina de Minas Gerais, Minas 
Gerais, v. 21, n. 1, p.32-41, fev. 2011.

OLIVEIRA, Jose Egidio Paulo; VENCIO, Sergio (org). Diretrizes da Sociedade Brasileira de Diabetes: 2013-2014/ Sociedade Brasileira de Diabetes. São Paulo: Ac. farmaceutica, 2014.

PADILHA, Patricia de Carvalho et al. Terapia nutricional no diabetes gestacional: Nutritional therapy in gestational diabetes. Revista de Nutrição, Campinas, v. 23, n.1, p.95-105, fev. 2010.

QUEIROZ, Pedrita Mirella Albuquerque. Nutritional profile and associated factors in women with gestational diabetes. Nutr. clin. diet. hosp, [s.I.], n. 2, p.96-102, maio 2016. SEDCA.

SCHMITT ML, Ribeiro SL, Paes MAS, Ribeiro RM. Prevalência de diabetes gestacional no município de São Joaquim - SC. RBAC 2009;41(1): 43-45.

SOUSA, Vivian Braga Gomes de et al. Gestação e diabetes: Relação entre estado nutricional e o controle glicêmico. Revista Brasileira de Promoção a Saúde, Fortaleza, v. 27, n. 4, p.541549, dez. 2014.

WEINERT, Leticia Schwerz et al. Diabetes gestacional: Um algoritmo de tratamento multidisciplinar. Arq Bras Endocrinal Metab., Porto Alegre, v. 7, n. 55, p.435-445, out. 2011. 